\title{
Technological interventions to reduce loneliness and social isolation among community-living older adults: A scoping review
}

\author{
Andrew Wister $\mathrm{PhD}^{\mathrm{a}, \mathrm{b}, \mathrm{c}, *}$, Eireann $\mathrm{O}^{\prime}$ Dea $\mathrm{MA}^{\mathrm{a}}$, Ian Fyffe $\mathrm{MA}^{\mathrm{a}}$, Theodore D. Cosco \\ $\mathrm{PhD}^{\mathrm{a}, \mathrm{b}, \mathrm{d}}$ \\ ${ }^{a}$ Gerontology Research Center, Simon Fraser University, 2800-515 West Hastings St. Van- \\ couver, Canada; ${ }^{b}$ Gerontology Department, Simon Fraser University, 2800-515 West \\ Hastings St. Vancouver, Canada; 'Institute on Aging and Lifelong Health, University of \\ Victoria, Victoria, Canada; ${ }^{d}$ Oxford Institute of Population Ageing, University of Oxford, \\ Oxford, UK; *Corresponding author: wister@sfu.ca
}

\begin{abstract}
Background: Loneliness and social isolation are recognized as significant challenges affecting older adults living in the community. In recent years, there has been an increase in the use of technological interventions to address these issues.

Objective: The primary objective of this study is to provide a comprehensive overview of the types of technological interventions that have been developed to reduce loneliness and/or social isolation for community-dwelling older adults. The secondary objective is to compare via mapping the technological intervention article characteristics in terms of date of publication, country of publication, study design, sample characteristics, loneliness/ social isolation measure, and efficacy/support for the intervention.

Method: Using the Joanna Briggs Institute (JBI) methodology (Joanna Briggs Institute, 2011) and the PRISMA extension for systematic/scoping reviews, a scoping review of seven academic databases (Academic Search Premier, Ageline, Global Health, MEDLINE, PsycINFO, Web of Science and ClinicalTrials.gov) was conducted.

Results: This scoping review identified 26 technological approaches to reducing loneliness and social isolation that met our criteria. We found three groupings; (1) computer and tablet-based competence training; (2) health-oriented technical interventions; and 3) Video games and animatronic pets interventions. The majority of technological interventions (15 out of 26) were effective in demonstrating support for reductions in loneliness and/or social isolation among older adults. Issues of accessibility, technology literacy, and complexity of the intervention were found to act as barriers to uptake.

Conclusion: A variety of technologies have been employed to reduce social and loneliness amongst older adults, with a growing body of evidence in support of technologydriven interventions.
\end{abstract}

Keywords: scoping review, technology, loneliness, social isolation, older adults

\section{INTRODUCTION}

Increasingly prevalent among community-dwelling older adults, loneliness and social isolation are recognized as significant international public health concerns (Fakoya, McCorry \& Donnelly, 2020). These concepts are unique yet interrelated with multiple definitions, conceptualizations, and measurements in the literature (Courtin \& Knapp, 2017; National Seniors Council, 2014a; 2014b; Nicholson, 2009; Toepoel, 2013; Valtorta et al., 2016; Weldrick \& Grenier, 2018). Loneliness is defined as the subjective perception that intimate and social needs are not being met. Whereas social isolation refers to a multifaceted objective condition in which there is an absence of social connectedness within the family, friendship, and community social networks (Newall \& Menec, 2019; Wister, Menec \& Mugford, 2018).
While the prevalence of these conditions varies, it has been estimated that between one-third and one-quarter of older adults will experience loneliness and/or social isolation; about 10 percent will experience chronic levels of these conditions (Fakoya, McCorry \& Donnelly, 2020; Grenade \& Boldy, 2008; Victor, et al., 2005; Weldrick \& Grenier, 2018).

Loneliness and social isolation are associated with higher rates of mortality, compromised physical health status, poorer health behaviours, and lower levels of mental health and psychological well-being, including depression, anxiety, lower life satisfaction, and happiness (Courtin \& Knapp, 2017; Golden et al., 2009; Leigh-Hunt, Bagguley, Turner et al., 2017; Wister, Cosco, Mitchell et al., 2019). Furthermore, these social- 


\section{Technology, loneliness, and social isolation}

psychological contexts have been associated with poorer access to health care services, and lower health care utilization (Newall, McArthur, \& Menec, 2015). In response to this growing public health problem, community, health care, and long-term care organizations that serve older adults have developed a spectrum of programs that aim to reduce levels of loneliness and social isolation using technologically-driven solutions (Baker et al., 2018; Chen \& Schulz, 2016; Fields et al., 2020; Wister \& Kadowaki, forthcoming).

Several gaps in this literature compromise the translation of knowledge gained from technological approaches to loneliness and social isolation interventions. Most of these programs have not been rigorously evaluated for efficacy either under ideal conditions, or effectiveness of the intervention in terms of application in natural settings. Also, multifactorial design and statistical challenges make it difficult to isolate the 'technology effect' from the 'human effect' for interventions that combine these elements. Additionally, although rates of technology use have risen significantly over time, approaching 75 percent among older adults (Pew Research Center, 2017), a 'digital divide' remains for some older adults that prevent them from affording or accessing technology, or not having the technological literacy to use them effectively (Gonzales, 2016; Yu et al., 2016)). We seize the opportunity to conduct a scoping review to: (a) identify technological approaches to reducing loneliness and social isolation that show promise; and (b) provide a foundation for subsequent higher-order reviews. Our objectives justify the use of a scoping review, which frequently serves as a method of identifying the types of evidence available as well as gaps in knowledge in a field (Munn et al., 2018).

The relevance of studying loneliness and social isolation among older adults has been magnified during the COVID-19 pandemic. The pandemic has exacerbated loneliness and social isolation, likely due to increased susceptibility and seriousness of viral risk (Kotowal et al., 2021; Shahid et al., 2020), combined with physical and social distancing (especially stay-at-home policies) (Wister \& Speechley, 2020). Most of the longitudinal studies comparing levels of loneliness pre and peri-pandemic have reported higher levels of loneliness during the beginning periods of the pandemic (Kotowal et al., 2021; Stoltz et al., 2021); however, some studies show a plateauing or even decrease over the course of the pandemic (Choi et al., 2021; Luchetti et al., 2020). Evidence for the impact of the pandemic on social isolation has been equivocal (Herrera et al., 2021; Kotwal et al., 2021; Strutt et al., 2021). Policies related to viral transmission miti- gation policies indicate potential importance of technology-driven interventions to address loneliness and social isolation during the COVID-19 pandemic, such as telephone befriending, digital applications, virtual support groups, educational approaches (Conroy et al., 2020; Cosco et al., 2021; Daly et al., 2021; Smith et al., 2021; Xie et al., 2021). However, these approaches have not been extensively evaluated during the pandemic. While further study is needed, risk, response, and resilience associated with pandemic point to potential areas in which technological applications may significantly reduce loneliness and social isolation, especially the most vulnerable and those with pre-existing conditions or multiple COVID-19 risk factors.

Technology has the potential to play a critical role in supporting community-dwelling older adults' ability to maintain healthy levels of social connectedness and engagement by providing a substitute for face-to-face social interaction or complementing human contact with technological innovation. At the conceptual level, technological approaches to loneliness and social isolation can be understood from a resilience model of aging. Resilience has been defined in a number of ways (Cosco et al., 2019; Wister \& Cosco, 2020), but is commonly defined as the ability to adapt and thrive when faced with one or more adversities (Klasa et al., 2021; Wister et al., 2016). According to Infurna (2021:808) there are six core elements of resilience: "(1) exposure to risk or adversity, (2) the response or manifestation of positive adaptation despite encountering risk or adversity, (3) individual variations surrounding the response to risk or adversity, (4) protective factors that predict positive adaptation, (5) resilience is a dynamic process that requires a methodology to match this notion (e.g., use of contemporary methods of analysis and longitudinal data), and (6) resilience is a multidimensional construct." A resilience and aging model has been used to understand processes of adaptation and recovery that are embedded within nested socio-ecological domains (e.g., individual, family, neighbourhood, municipality, health region, province, country, global levels). Given that loneliness and social isolation have been established as forms of a serious challenge for many older adults, technological interventions can be utilized to foster access to social support, social connectedness, information, and other types of resources that enhance the ability of individuals, families and structural systems to bounce back from these forms of social adversity. For community organizations, health and long-term care systems that target less resilient and vulnerable socially isolated older adults, technological innovations may enrich their outreach programs in a number of ways, including diffusion of tech- 
nological communication devices, training programs, improve efficiencies, and build capacity. While there have been a few scoping reviews that focus on technological solutions aimed at loneliness and social isolation (Baker et al., 2018; Chen \& Schulz, 2016), these have focused on social isolation, and they have not incorporated new studies that have been published.

\section{Study objectives and review question}

The primary objective of this scoping review is to provide a comprehensive overview of the types of technological interventions that have been developed to reduce loneliness and/or social isolation for community-dwelling older adults. The secondary objective is to compare via mapping the technological intervention article characteristics, date of publication, country of publication study design, sample characteristics, loneliness/ social isolation outcome measure, and efficacy/ support for the intervention.

\section{Methods}

The study objectives, methods, and inclusion/ exclusion criteria were outlined in advance and documented in a scoping review protocol (Wister et al., 2021). This scoping review was comprised of studies with participants aged 60 or older, or with a mean age of 65 and above. This expanded age criterion was needed in order to include potentially relevant articles in this limited area of research. Similar approaches have been utilized in past scoping reviews (Cheng et al., 2020). This review considered any type of study that utilized technology as a means to diminish levels of the outcome variables loneliness and/or social isolation, and included quantitative, qualitative, and mixed methods studies. This review follows the Joanna Briggs Institute (Joanna Briggs Institute, 2011) methodology and the PRISMA extension for systematic/scoping reviews. It was developed by three researchers including an academic professor, a doctoral university student, and a research assistant. The doctoral university student and the research assistant served as the main reviewers.

\section{Search strategy}

A three-stage search strategy recommended by the JBI was utilized. The first stage entailed generating a list of search terms based on the titles and abstracts of relevant articles. The initial search terms were applied in seven databases: Academic Search Premier, Ageline, Global Health, MEDLINE, PsycINFO, Web of Science, and ClinicalTrials.gov. In order to obtain results with our outcome criteria, these search terms included loneliness or social isolation in conjunction with the search terms: older adults, elderly, program, evaluation, trial, intervention, computer, ICT, technology, internet, or robot. Spe- cific subject headings (ie: MeSH terms) were also used for certain databases (ie: Medline). The full search strategy is presented in Appendix I. All studies published in English up until September 2020 were included in the search parameters to be as inclusive as possible.

\section{Inclusion and exclusion criteria}

Inclusion and exclusion criteria were applied to the selected articles. An article was included if it: (1) included a population of subjects of at least 60 years of age or with a mean of age of $65+$, (2) had an outcome variable of loneliness and/ or social isolation, (3) focused on communitydwelling older adults, (4) focused on technological interventions for loneliness and/or social isolation, and, (5) was published in the English language. Technological interventions on adults aged younger than 60 years were excluded; however, for studies where only a mean age was reported, mean age of 65 was considered sufficient. Institutionalized populations were also excluded, as were blended populations of both institutionalized and community-dwelling persons. In the third stage, additional sources were found by hand-searching the reference lists of articles.

\section{Study selection, organization, and reviewing results} In order to expedite the construction of a bibliography, Zotero - a bibliographic reference management tool - was used. Zotero was employed in order to organize and screen all articles. Sources that met the inclusion criteria were uploaded into Zotero and duplicates were excluded. The review process included two stages of screening performed by two independent reviewers. The first screening stage was a title and abstract review for appropriateness. The second screening stage was a full-text review for inclusion or exclusion. Reasons for exclusion have been noted and presented in a PRISMA flow diagram presented below (Figure 1).

The data analysis software NVivo 12 was utilized to extract data from selected articles during the full-text review process. This extracted data from the articles were inputted into the data extraction tool. The data extraction tool was created with input from the entire team. The data extraction tool was generated by using the JBI Reviewer's Manual Appendix 11.1 (Joanna Briggs Institute, 2011) as a template. Inclusion/Exclusion Criteria will include population (older adults aged 60 or older or with a mean age of 65 and above vs not), concept 1 (technological intervention vs not), concept 2 (loneliness and/or social isolation as outcomes vs not), context (community vs institution) and types of evidence source (academic journal article vs grey literature vs thesis or dissertation). The Evidence Source Details and Characteristics categories will include citation 


\section{Technology, loneliness, and social isolation}
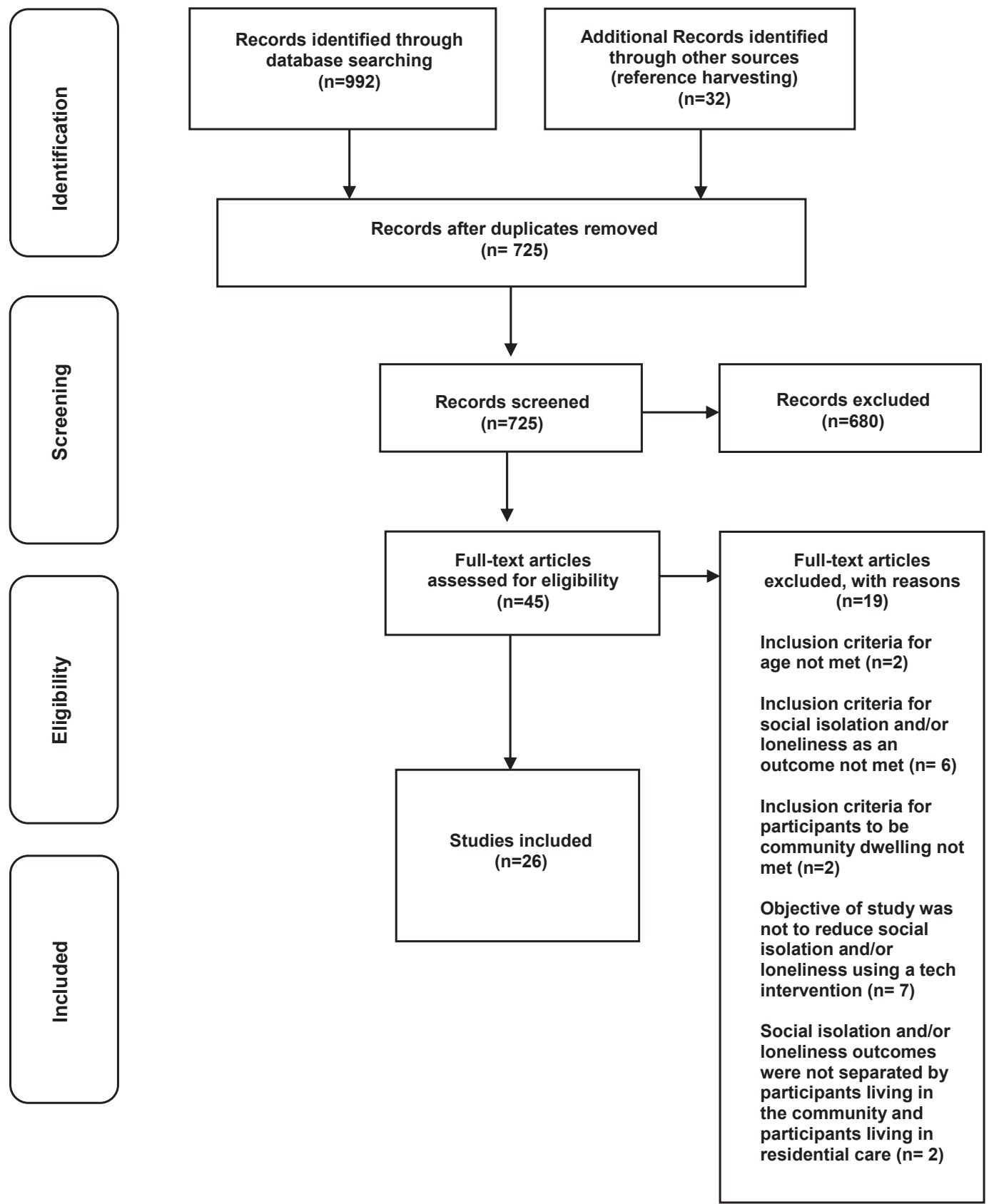

Figure 1. PRISMA flow diagram illustrating the search strategy

details (e.g. author(s), date, title, journal, volume, issue, pages), country, context (type of technological intervention), participants (details e.g. age/sex and number). Details/Results extracted from the source of evidence section included design (e.g. experimental design, qualitative), outcome (loneliness or social isolation), tool (e.g. scale used to assess loneliness or social isolation), and type (individual or group intervention). Due to the comprehensive nature of the data extraction tool, alterations were not required.
To determine whether the independent researchers were utilizing an approach that was consistent with the purpose and research question, the first twenty studies were reviewed and discussed. This strategy was consistent with that which has been articulated by Levac et al., (2010). This step ensured early calibration and correction of any systematic patterns of discrepancies that may have arisen between reviewers and to determine whether the instructions for screening are sufficient. Subsequently, eligibility was assessed with 
full-text screening by two independent reviewers. If the relevance of an abstract was unclear, full-text screening was conducted (Arksey \& O'Malley, 2005). The second round of calibration between reviewers occurred at this stage during full-text screening to ensure consistent data extraction. Any disagreements were resolved by consensus and a third reviewer was to be consulted to decide if consensus was not achieved initially.

\section{Data analysis and presentation}

Information from the data extraction tool has been presented in tables for visualization, transparency, and clarity. These tables include country, year of publication, study design (including n size, measure, study design, and whether the group was an individual or group intervention), and technological intervention.

\section{Literature search}

Electronic database searches identified a total of 992 articles. A hand-search was then conducted using the reference lists of systematic review articles identified prior to the electronic database search. This produced an additional 17 articles for a total of 1009 articles. Duplicates were then removed, which left a total of 710 articles. This was followed by additional reference harvesting, this time the systematic reviews identified during the electronic database search. This resulted in a total of 15 additional articles (for a total of 32 articles found through reference harvesting), bringing the total number of articles for abstract review to 725 . An abstract review of these 725 articles was then conducted, after which 45 articles were included and 680 excluded. The full text of 45 articles was assessed for eligibility, with 19 being found not to meet study criteria, resulting in 26 being included in this scoping review. The characteristics of these 26 included articles are discussed below.

\section{Results \\ Study characteristics}

A summary table of each study showing year of publication, sample size, age (mean and standard deviation where available; or $60+$ range level), gender and ethnicity of study populations, loneliness and/or social isolation measures, study design, type of technological intervention, intervention target (group vs. individual), and support/efficacy is presented below (Table 1).

\section{Year of publication}

The included studies were published between 2005 to 2020, with 5 articles published in the year 2020 alone (Table 1). Studies were conducted in 10 countries (Europe combined), primarily in the United States of America $(n=13)$ and Europe $(n=9)$, with Australia, the United Kingdom,
Canada, and Asia having one study each. One study took place in two countries (Slovenia and Finland) and compared the results.

\section{Age of study participants}

Twenty-two studies reported a mean age of 65 and above for participants, while four studies included participants who were aged 60 and above.

\section{Sample size}

The sample sizes for the included studies ranged from 4 (Ballantyne et al., 2010) to 300 participants (Czaja et al., 2018). This is relevant for consideration of statistical power for quantitative studies and generalizability.

\section{Gender and ethnicity}

Twenty-three included studies had study populations that were over $50 \%$ female, two included populations that were $>50 \%$ male, and one study did not report on sex. The majority of studies included (16) did not report on the ethnicity or race of study participants. Of the studies that did, 8 contained populations that were $>50 \%$ White or Caucasian, while two had populations that were $<50 \%$ non-White (in both cases, the majority of study participants were African American).

\section{Measures}

Various instruments were used to measure loneliness and/or social isolation for each technological intervention study. The majority of the studies included in this scoping review (20 out of 26) measured changes in loneliness outcomes among older adult participants. Several of these studies utilized versions of the UCLA loneliness scale, including the Revised UCLA Loneliness Scale (Russell et al., 1980), the UCLA Loneliness Scale (Version 3) (Russell, 1996), and the Three-Item UCLA Loneliness Scale (Hughes et al., 2004). The de Jong Gierveld Loneliness Scale (de Jong Gierveld \& Kamphuis, 1985) was also frequently used. Additional instruments used to measure loneliness included the 6-item de Jong Gierveld Loneliness Scale (de Jong Gierveld \& Van Tilburg, 2006), and the 8-item PROMIS (Patient-Reported Outcomes Measurement Information System) Social Isolation Scale (PROMISL) (Riley et al., 2011). Changes in social isolation outcomes among participants were measured using instruments including the 4-item Social Interaction Subscale of the Duke Social Support Index (DSSI-I) (Landerman, George, Campbell, \& Blazer, 1989), and the Patient Reported Outcome Measurement Information System (PROMIS)- Social Isolation (6-item) (Riley et al., 2011.). Openended and semi-structured interviews, as well as self-report questionnaires, were also utilized to measure loneliness and/or social isolation in a small number of studies $(\mathrm{n}=$ ?). 


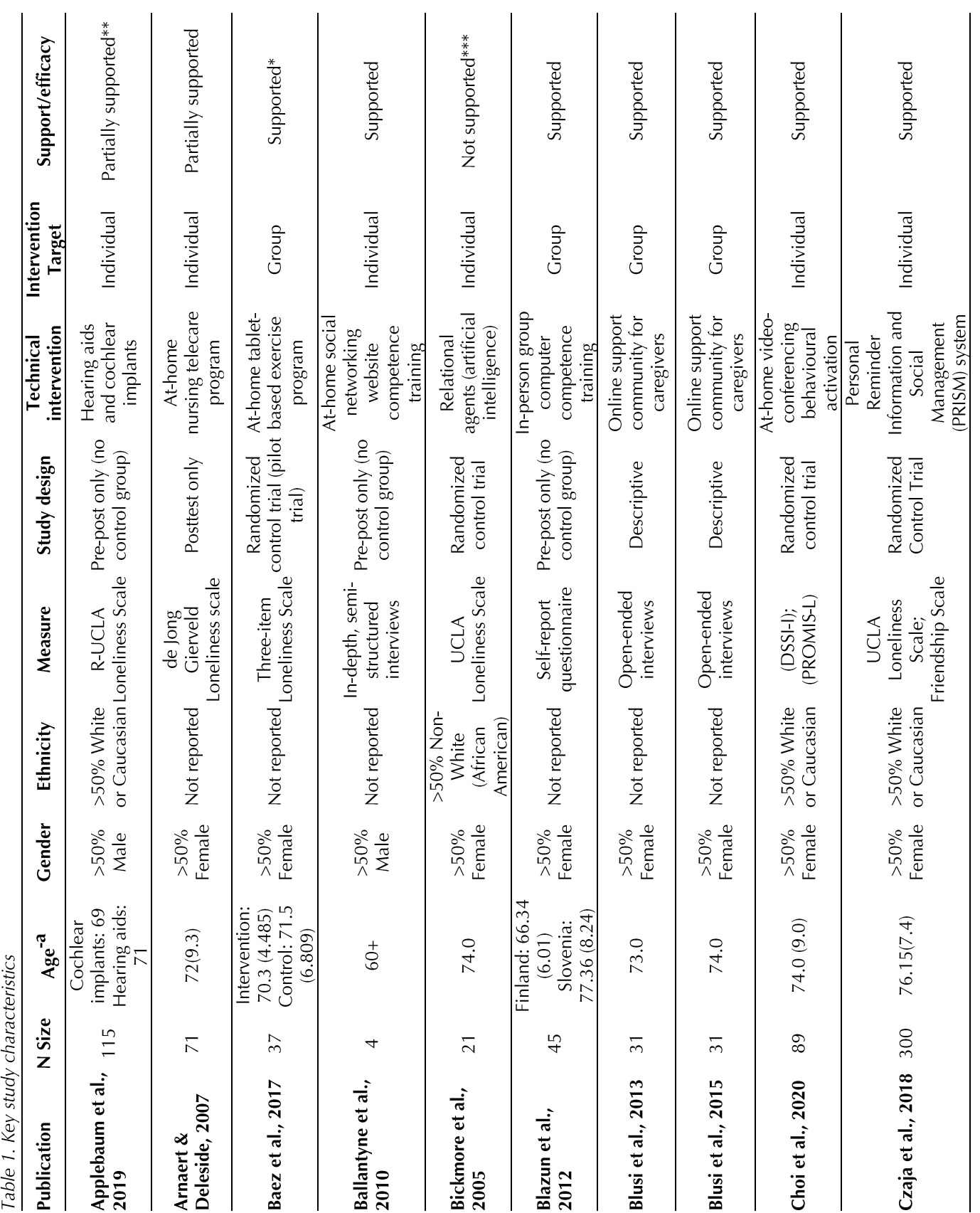

Study methodology/Design

Examples of pre-posttest study designs included Applebaum et al., (2019), in which levels of loneliness were measured among study participants prior to (baseline) the insertion of either a hearing aid or cochlear implant device, and then at 6-month, 1-year, and 5-year increments. Using qualitative methods, Ballantyne et al., (2010), conducted in-depth interviews with participants before and after their participation in a social networking website. Neil-Sztramko et al. (2020) collected data on self-reported social isolation and loneliness at baseline and a month following 


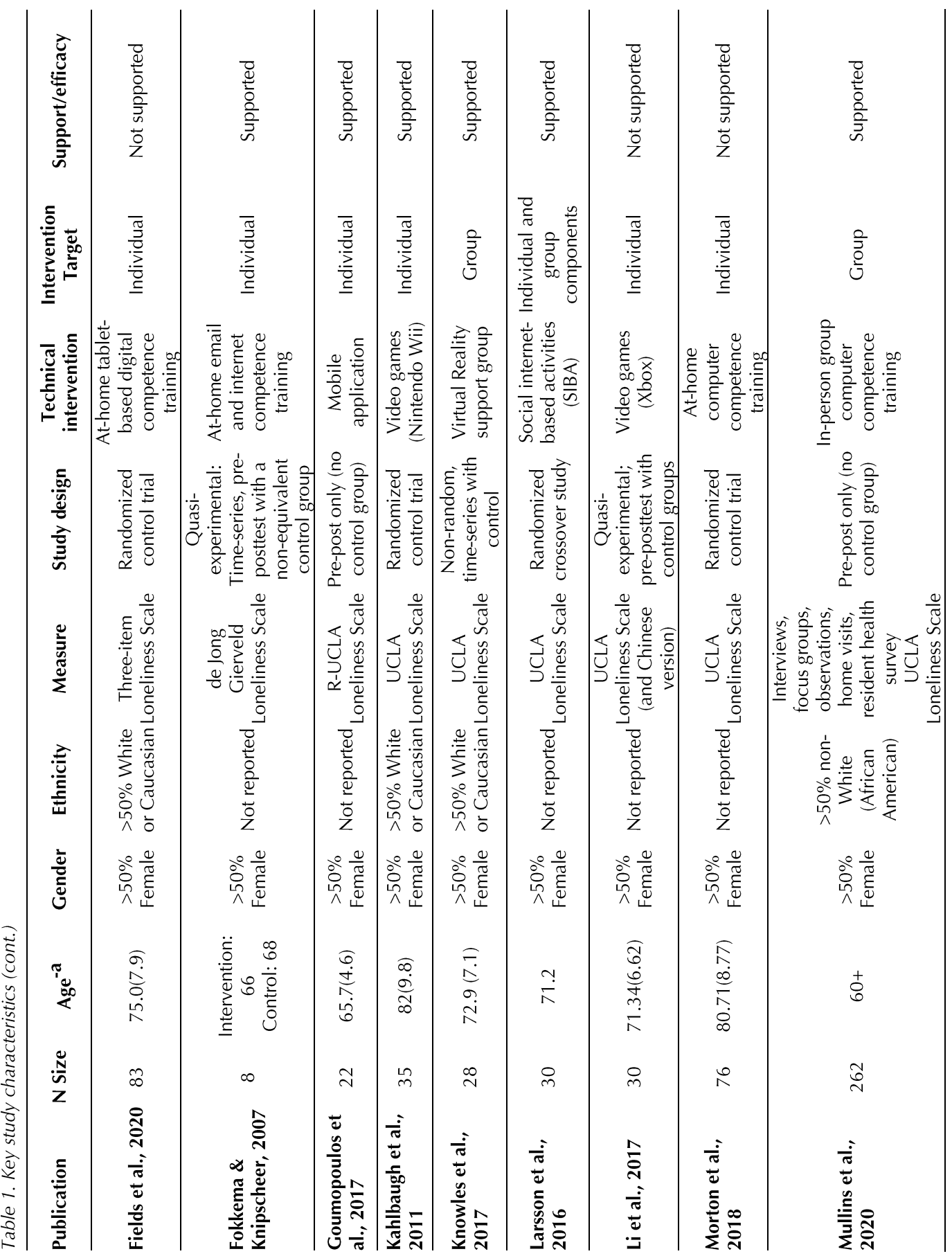

participation in a 6-week workshop program (no control group) during which older adults learned to use various features of an iPad and the internet. Other studies with a pre-posttest study design included Blazun et al., (2012); Mullins et al., (2020); Goumopoulos et al., (2017), and Van der Heide et al., (2012). Anaert \& Deleside (2007) utilized a posttest-only design, in which levels of functioning (ie: loneliness) were measured at three 6 -month intervals following a video-conferencing intervention.

Tomasino et al., (2017) and Li et al., (2017) utilized quasi-experimental designs, in which a pre-post-test design was used with a non-randomized control group. In Tomasino et al. (2017), a pre-specified group assignment was performed, and participants were assigned to either receiv- 


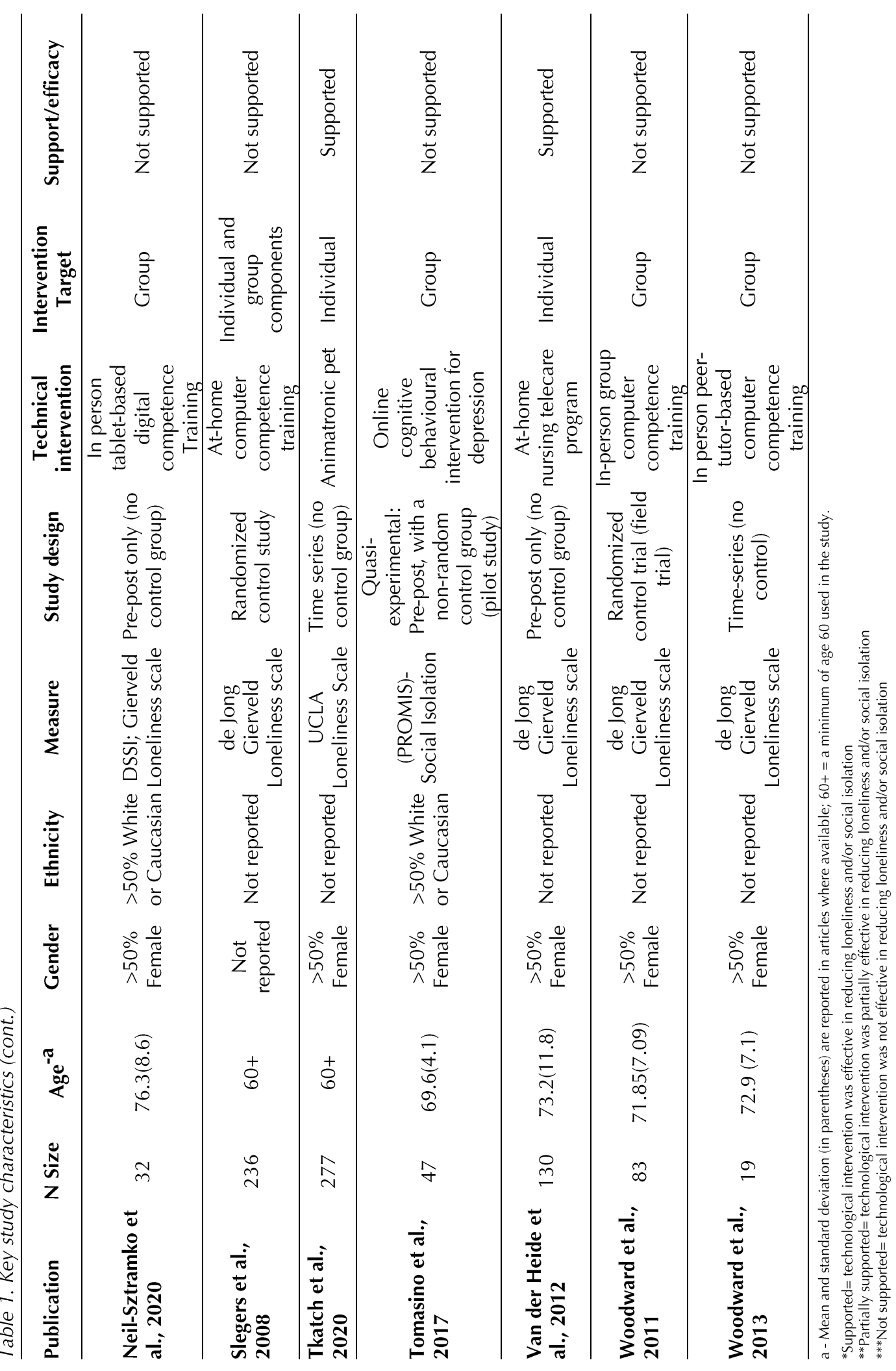


ing either the individual internet intervention, the internet intervention with peer support, or to a wait-list control group (who eventually received access to the individual internet intervention following the waiting period). Assessments were administered at baseline and after 8 weeks of receiving the intervention. Li et al., (2017) assigned participants to three potential groups: Exergames (exercise video games), traditional exercise, or care-as-usual. Loneliness measures were assessed at baseline and after six weeks of receiving the intervention.

Studies using randomized control trial study designs included Bickmore et al., (2005) and Choi et al., (2020). Bickmore et al. (2005) compared loneliness scores between participants who engaged in an online exercise program (which included a relational agent) for two months with participants randomized to receive standard care. Choi et al., (2020) randomized a group of 89 participants to either receive five weekly video conference sessions or the active control intervention (friendly visits). One study that differed slightly from a typical randomized control trial but still included within this category is Larsson et al. (2016), who conducted a randomized crossover study. After three months of receiving the technological intervention (social internet-based activities) participants crossed over to the control group for an additional three months, while participants initially in the control group went through the same process in reverse. Measurements of loneliness were conducted prior to the intervention (T1), after the first three months (T2), and following the final intervention and control period (T3). Other studies that used a randomized control trial design included Baez et al., (2017); Czeja et al., (2018); Fields et al., (2020); Kahlbaugh et al., (2011); Morton et al., (2018), Slegers et al., (2008); and Woodward et al., (2011).

Fokkema \& Knipscheer (2007), Knowles et al. (2017), Tkatch et al., (2020), and Woodward (2013) employed a time-series design. Fokkema \& Knipscheer (2007) and Knowles et al., (2017) utilized quasi-experimental approaches, in which participants were assessed at multiple time points, and their loneliness scores at baseline and post-measurement were then compared to a non-randomized control group (no intervention). Knowles et al. (2017) measured loneliness scores among participants in virtual reality grief support groups at three-time points (baseline, post-intervention, and two months post-intervention), and subsequently compared results to participants in an active, non-randomized control group (grief website). Tkatch et al. (2020) administered surveys measuring loneliness to participants at baseline, 30 days, and 60 days after they had received an animatronic pet with no control group. Similarly, Woodward et al., (2013) collected loneliness and social isolation scores from participants in a peer-tutor computer training program at baseline, three months, six months, and nine months, with no control group. Finally, Blusi et al., (2013, 2015), used descriptive designs to assess the impact of an ICTbased support program for rural caregivers on loneliness (Blusi et al., 2013) and social isolation (Blusi et al., 2013. 2015). Following recruitment, participants were instructed to use the technical equipment provided, and following this engaged in open-ended interviews after 1 year (Blusi et al., 2013) and 1.5 years (Blusi et al., 2015).

\section{Group vs. individual targeted intervention}

The technological interventions used to reduce loneliness and/or social isolation among older adult participants also varied in terms of whether they were administered as a group or individual intervention. "Group" interventions provided individuals the opportunity to interact with other participants who were also receiving the same intervention, either virtually using a tablet or computer program, or in-person in the context of a classroom. Examples of group interventions are highlighted in studies by Baez et al. (2017), in which 37 older adults participated in a personalized group exercise program for eight weeks using a tablet, which enabled participants to "see each other" as avatars in the virtual classroom, and Neil-Sztramko et al., (2020), in which participants attended six weekly workshops covering the basic features of a tablet. By contrast, "individual" interventions were less social in nature, with participants engaging in the intervention alone or potentially alongside a study researcher. An example of such an intervention is detailed in Morton et al. (2018), in which older adult participants were given a computer and broadband infrastructure to install in their homes. "Care Technologists" administered training, and participants kept the equipment for 12 months, at which time changes in loneliness were assessed. Two included studies administered both individual and group interventions to participants. One example of this was Slegers et al., (2008), in which participants first participated in computer training classes, and following that, were randomized to either an intervention or control group. Those in the intervention group received a personal computer as well as broadband internet connection, and then used the computer for their own needs for a period of one year with no social element.

Support for/efficacy of technological interventions This scoping review has been conducted in order to provide a comprehensive overview of the types of technological interventions that have been developed to reduce loneliness and/or social isolation for community-dwelling older adults. Due to 
the heterogeneity of the exposures and outcomes in the included studies, meta-analyses were not possible; therefore, narrative syntheses of study findings have been conducted (Table 1). A general description of the efficacy findings (supported, partially supported, not supported) based on the reported evidence in each article pertaining to the technological intervention on loneliness and/ or social isolation. In this case, a study that has been "supported" indicates that the intervention in question was effective in reducing loneliness and/or social isolation according to the authors' conclusions. "Partially supported" indicates that the intervention was partially but not fully effective in reducing loneliness and/or social isolation based on an assessment of the study results (e.g. loneliness decreased initially following baseline but increased at follow-up, loneliness decreased but not social isolation, etc.). Finally, a "not supported," intervention indicates that the intervention was reported to not affect reducing loneliness and/or social isolation among study participants. Of the 26 included studies, 15 contained supported/effective interventions. Two studies contained interventions that were partially supported, and 9 studies contained studies that were not supportive.

\section{Technological interventions}

A wide variety of technological interventions to address loneliness and/or social isolation among older adults were reviewed, which have been separated into three categories: (1) Computer and tablet-based competence training to reduce loneliness and/or social isolation among older adults, (2) Physical and mental health-oriented interventions to reduce loneliness and /or social isolation among older adults, and (3) Video games and animatronic pets to reduce loneliness and/or social isolation among older adults.

Computer and tablet-based competence training to reduce loneliness and/or social isolation among older adults

A total of 10 out of the 26 studies reviewed focused on technological interventions in which participants were provided with a computer and tablet-based competency training. The goal of these interventions was to increase older adults comfort with using technology, and to enable them to eventually utilize the internet, social networking websites, and other tools to communicate with others, and thus decrease their levels of loneliness and/or social isolation. Components of these interventions included learning basic computer and tablet skills (turning the computer or tablet on and off, using a keyboard and mouse, become familiar with certain applications, how to use a touch screen), how to write documents, how to use the internet and search for information, how to use email, online safety, online shopping, social media instruction, as well as communicating with others via applications such as FaceTime and Skype. These interventions varied in terms of delivery method, with 5 including in-person classroom instruction (Blazun et al., 2012; Neil-Sztramko et al., 2020; Mullins et al., 2020; Woodward et al., 2011; Woodward et al., 2013) and 5 not including any kind of classroom instruction, but rather one-onone training sessions, typically in the participants' home (Ballantyne et al., 2010; Fields et al., 2020; Fokkema \& Knipscheer, 2007; Morton et. al, 2018; Slegers et al., 2008).

Health-oriented technological interventions to reduce loneliness and/or social isolation

The most common type of technological intervention (12 out of 26) fell under the broad category of health-oriented technological interventions to reduce loneliness and/or social isolation. These interventions either sought to decrease levels of loneliness and/or social isolation while also providing an intervention to increase physical mental, or social health (e.g. exercise programs, nursing care, hearing aids/cochlear implants), or, in the case of a select few interventions, targeted a specific physical, mental, or social health problem (e.g., depression, physical fitness, grief, small social network/lack of social support), while also measuring changes in loneliness and/or social isolation as a secondary outcome. The technological interventions in this category included nursing telecare programs (Arnaert \& Delesie, 2007; van der Heide et al., 2012), in which older adult participants were able to have access to nursing care and support through video-conferencing technology from their own homes. In the case of the intervention "CareTV," (van der Heide et al., 2012), participants were also able to use the program to contact family and friends, as well as a welfare organization with any questions regarding housing and welfare. Three technological interventions sought to improve physical health outcomes among older adults while also targeting loneliness. Baez et al. (2017) provided tablets to older adult participants for engagement in a group fitness class, while Applebaum et al. (2019) provided hearing aids and cochlear implants to older adults and assessed changes in loneliness over the course of five years (Applebaum et al., 2019). Bickmore et al., (2005) utilized relational agents, computational artifacts designed to build and maintain socialemotional relationships to act as exercise advisors for older adults participating in a computer program called FitTrack. In this intervention, the relational agents interacted with participants in the form of software humanoid animated agents, building social-emotional relationships over time and helping them to stay on track with regards to their physical activity goals. Two interventions utilized forms of behavioural therapy: behav- 
ioural activation (Choi et al., 2020) and cognitive behavioural therapy (Tomasino et al., 2017) to target loneliness and/or social isolation among older adult participants. Choi et al., (2020) utilized a lay-coach facilitated video-conferencing programs to increase social interaction and decrease feelings of loneliness among older adult participants, while Tomasino et al., (2017) used MoodTech, an 8-week online intervention based on Cognitive Behavioural Therapy principles to target depressive symptoms, with social isolation as a secondary outcome. Knowles et al. (2017) also targeted mental health outcomes (including grief, depression, perceived stress) as well as loneliness among older adult widow(ers) using a virtual reality support group.

Several interventions aimed to improve participants' social health (ie: number of meaningful relationships, positive interactions with others) by enhancing their social networks, while also reducing loneliness and social isolation. In a study by Larsson et al., (2016) occupational therapists administered an intervention in which older adults (who were already familiar with using the internet and social networking) participated in Social Internet-Based Activities (SIBAs). Their progress and various goals were monitored by the occupational therapists via group meetings, individual meetings, and at-home support. Blusi et al. $(2013,2015)$ provided internet communication technology (ICT)-based caregiver support (ACTION) to older adult family caregivers living in rural areas of Sweden, allowing individuals to access a secure social community of other family carers, call centres, and educational programs related to caregiving. Goumopoulos et al., (2017) developed a type of Ambient Assistive Living system in the form of a mobile application called Senior App suite, which provided older adults with information and programs related to service access, social networking, and emergency detection to increase feelings of social inclusion. Similarly, Czeja et al., (2018) designed a specialized computer system called PRISM (Personal Reminder Information and Social Management), which provided participants access to the internet, a resource guide, an online classroom, email, games, social networking, etc., to increase social support and connectivity.

Video games and animatronic pets to reduce loneliness and/or social isolation

The final category includes interventions that utilized video games and robotic technology to target loneliness and/or social isolation among older adults. Both Li (et al., 2017) and Kahlbaugh et al., (2011) assessed the impact of exercisebased video games on feelings of loneliness among participants. Li et al., (2017) evaluated "Exergames" developed specifically for older adults on the Xbox Kinect platform, which included Skiing, Hiking, Pikkuli, Chinatown Race, and RehaMed Volleyball. Kalhnaugh et al. (2011) provided their participants with a Nintendo Wii console to engage in Wii bowling. Tkatch et al., (2020) utilized robotic technology to simulate social connections and decrease loneliness for older adult participants. Participants were provided with an animatronic pet (cat or dog) and were instructed to treat it like a pet for 60 days.

\section{Discussion}

The primary objective of this scoping review was to provide a comprehensive overview of the types of technological interventions that have been developed to reduce loneliness and/or social isolation for community-dwelling older adults. The 26 included studies reviewed demonstrate a wide array of technological approaches that have been used to diminish levels of loneliness and social isolation among community-living older adults. These ranged from animatronic pets to artificial intelligence, to at-home computer competence training, to at-home video conferencing, to social network website training, to video games, and beyond. The most common technological intervention (12 out of 26) described health-oriented technological interventions to reduce loneliness and/or social isolation, in which both participants' mental, physical and social health, as well as levels of loneliness, were affected. Most studies (20 out of 26) measured changes in loneliness, while 2 studied only changes in social isolation, and 4 assessed both loneliness and social isolation. It should also be noted that some technologies, such as automated pets, are targeted at older adults living in institutional environments rather than the community.

While some of the more complex technological innovations, such as artificial intelligence, maybe inaccessible to the average community-dwelling older adult; several approaches utilized by the interventions were more accessible. For example, the usage of video conferencing technology seems to be a relatively easy and inexpensive method for community-dwelling older adults to connect with existing social networks and reduce levels of loneliness and social isolation. Indeed, since workplaces have utilized this technology during the COVID-19 pandemic in order to replace or support in person social interaction; it is likely that older adults in the community would benefit from this as well. Furthermore, low-tech approaches such as the telephone were found to be very useful during the pandemic to replace typical face-to-face programs and services, given social/physical distancing policies. Evaluation of these approaches and the ways in which community services were adapted to coincide with 
COVID-19 safety protocols may advance our understanding of technological innovations to address loneliness and social isolation among older adults. It is also important to consider the potential of such interventions to address other aspects of well-being beyond experiences of loneliness and/or social isolation for older adults. For example, interventions within the categories of computer and tablet-based competence training and health-oriented technological interventions also stand to benefit older adults by increasing their technological literacy and confidence, and increasing physical activity and mental health outcomes, respectively.

Our results coincide with other literature on this topic indicating that there is a spectrum of technologies that have been applied to loneliness and social isolation (see, Baker et al., 2018; Chen \& Schulz, 2016; Fakoya et al. 2020). Some of these are directly aimed at these outcomes, whereas others address them indirectly. The proliferation of technological interventions in this area will likely continue to grow, given the development and uptake in technologies generally, and the effects of the pandemic that may have exacerbated issues of loneliness and social isolation, or at least brought them to the forefront (Kotowal et al., 2021). Yet, the literature in this field is fragmented due to the selection of technological type, specific outcome of interest, and population under study. This makes the integration of research knowledge challenging until the body of literature in these sub-domains grows.

While our search strategy was focused on direct applications targeting loneliness and social isolation for older adults living in the community, many other relevant technological approaches need to be assessed in future work, including computer and internet training; social network site use (Facebook, Instagram); video-communication through videophones; telecare (video-telephone nursing care, care TV); web-based information, intervention and communication programs, etc.

In sum, our results highlight a variety of technological interventions aimed at reducing loneliness and social isolation among community-living older adults. However, the evidence to date remains equivocal, in part due to a fewer number of studies including social isolation outcomes, differential levels of support for outcomes, and the limited number of rigorous evaluation studies. For instance, studies using a quasi-experimental design (i.e., pre-post only design), can be prone to a placebo effect, and other threats to internal validity. For instance, it is difficult to separate effects associated with the technology on reducing loneliness and social isolation from placebo effects caused by treatment group participants' involvement in the study (Windsor, 20150. It should be noted that the assessment of intervention support in our scoping review was based on the reported findings and did not assess efficacy using more stringent criteria applied to randomized clinical trials (RCTs), such as effect size, statistical power, measurement, etc. Although assessment of these criteria was not the purpose of our scoping review, it remains for future research once a critical number of RCTs of interventions are available. It is expected that the results of this scoping review will provide insight into the next phase of systematic research on these outcomes.

\section{LimitATIONS}

Limitations to consider for this review include the lack of eligible studies conducted in lower technology-based locations such as Africa, and South America. Despite examining the experiences of older adults in multiple contexts (USA, Canada, United Kingdom, Australia, Sweden, Finland, Slovenia, The Netherlands, Italy, Greece, Singapore, and Belgium), the needs of older adults and the effectiveness of technological interventions in regions outside of Europe, North America, Asia, and Australia may differ and should be further examined. However, it should be noted that the English-only inclusion limitation of this scoping review was likely a contributing factor that limited the diversity of geographical contexts. Other limitations concern the fundamental nature of a scoping review itself, in which there is no formal evaluation of the quality of the evidence presented in the included studies, such as assessment of effect sizes, statistical power, or a meta-evaluation of results meeting a high threshold of statistical criteria.

\section{Conclusion}

This review has revealed that there are numerous types of technological interventions that can be used to address loneliness and/or social isolation among community-dwelling older adults. The majority of studies were conducted in the United States and Europe. Most (17 out of 26) utilized experimental approaches of either randomized control trials or pre-posttest study designs to assess the impact of technological interventions on reducing loneliness and/or social isolation among older adults. The interventions were dated from 2005 to as recent as 2020. While most studies included only measures of loneliness (20/26) with a range of reported support levels for technology effects, the supporting evidence for either loneliness or social isolation remains equivocal.

While some studies used common approaches in their interventions, others used highly specific types of technology (animatronic pets, relational agents) less commonly seen in the literature. 
Given that loneliness and social isolation continue to threaten the physical, mental, and social well-being of the growing aging population, it is important to consider the full spectrum of innovative methods of connecting older adults with others and to explore the potential of technology in this endeavor. Finally, as research studies become available based on the use of technologydriven interventions to reduce loneliness and social isolation during the COVID-19 pandemic, there will be opportunities to extend the knowledge base in this field of study.

\section{Funding}

TDC is a Michael Smith Foundation for Health Research Scholar (SCH-2020-0490) and receives funding from AGE-WELL Core Research Project (AWCRP-2020-03). TDC and AW's work is supported in part by funding from the Social Sciences and Humanities Research Council.

\section{References}

Applebaum, J., Hoyer, M., Betz, J., Lin, F. R., \& Goman, A. M. (2019). Long-term subjective loneliness in adults after hearing loss treatment. International journal of audiology, 58(8), 464-467.

Arnaert, A., \& Delesie, L. (2007). Effectiveness of videotelephone nursing care for the homebound elderly. Canadian Journal of Nursing Research Archive, 39(1), 20-36.

Baez, M., Far, I. K., Ibarra, F., Ferron, M., Didino, D., \& Casati, F. (2017). Effects of online group exercises for older adults on physical, psychological and social wellbeing: a randomized pilot trial. PeerJ, 5, e3150.

Baker, S. et al. (2018). Combatting social isolation and increasing social participation of older adults through the use of technology: a systematic review of existing evidence. Australasian Journal on Ageing, 37(3), 184-193.

Ballantyne, A., Trenwith, L., Zubrinich, S., \& Corlis, M. (2010). 'I feel less lonely': what older people say about participating in a social networking website. Quality in Ageing and Older Adults. 11(3), 25-35.

Bickmore, T. W., Caruso, L., Clough-Gorr, K., \& Heeren, T. (2005). 'It's just like you talk to a friend' relational agents for older adults. Interacting with Computers, 17(6), 711-735.

Blažun, H., Saranto, K., \& Rissanen, S. (2012). Impact of computer training courses on reduction of loneliness of older people in Finland and Slovenia. Computers in Human Behavior, 28(4), 1202-1212.

Blusi, M., Asplund, K., \& Jong, M. (2013). Older family carers in rural areas: experiences from using caregiver support services based on Information and Communication Technology (ICT). European journal of ageing, 10(3), 191-199.

Blusi, M., Kristiansen, L., \& Jong, M. (2015). Exploring the influence of Internet-based caregiver support on experiences of isolation for older spouse caregivers in rural areas: A qualitative interview study. International Journal of Older People Nursing, 10(3), 211-220.

Chen, Y.R.R. \& Schulz, P.J. (2016). The effect of information communication technology interventions on reducing social isolation in the elderly: a systematic review. Journal of Medical Internet Research, 18(1). DOI: 10.2196/jmir.4596.

Cheng, K. K. F., Siah, R. C. J., Ream, E., Kanesvaran, R., \& Armes, J. (2020). Protocol for a scoping review of digital health for older adults with cancer and their families. BMJ open, 10(8), e038876.

Choi, E. Y., Farina, M. P., Wu, Q., \& Ailshire, J. (2021). COVID-19 social distancing measures and loneliness among older adults. The Journals of Gerontology. Series B, Psychological Sciences and Social Sciences. https://doi.org/10.1093/geronb/gbab009

Choi, N. G., Pepin, R., Marti, C. N., Stevens, C. J., \& Bruce, M. L. (2020). Improving social connectedness for homebound older adults: randomized controlled trial of tele-delivered behavioral activation versus tele-delivered friendly visits. The American Journal of Geriatric Psychiatry, 28(7), 698-708.

Conroy, K. M., Krishnan, S., Mittelstaedt, S., \& Patel, S. S. (2020). Technological advancements to address elderly loneliness: Practical considerations and community resilience implications for COVID-19 pandemic. Working with Older People: Community Care Policy \& Practice, 24(4), 257-264. https:// doi.org/10.1108/WWOP-07-2020-0036

Cosco, T.D., Kok, A., Wister, A., \& Howse, K. (2019). Conceptualising and operationalising resilience in older adults. Health Psychology \& Behavioural Medicine, 7(1), 90-104.

Cosco, T.D., Fortuna, K., Wister, A., Riadi, I, Wagner, K., \& Sixsmith, A. (2021). COVID-19, social isolation, and the mental health of older adults: A digital catch-22. Journal of Medical Internet Research, 23(5), e21864-e21864.

Courtin, E. \& Knapp, M. (2017). Social isolation, loneliness, and health in old age: A scoping review. Health and Social Care in the Community. 25(3), 799-812. https://doi.org/10.1111/hsc.12311

Czaja, S.; Charness, N.; Fisk, A.; Hertzog, C.; Nair, S.; Rogers, W.; Sharit, J (2006). Factors predicting the use of technology: findings from the Center for Research and Education on Aging and Technology Enhancement (CREATE). Psychology and Aging, 21(2), 333-352.

Czaja, S. J., Boot, W. R., Charness, N., Rogers, W. A., \& Sharit, J. (2018). Improving social support for older adults through technology: Findings from the PRISM randomized controlled trial. The Gerontologist, 58(3), 467-477.

Daly, J. R., Depp, C., Graham, S. A., Jeste, D. V., Kim, H. C., Lee, E. E., \& Nebeker, C. (2021). Health Impacts of the Stay-at-Home Order on Community-Dwelling Older Adults and How Technologies May Help: Focus Group Study. JMIR Aging, 4(1), e25779. https://doi.org/10.2196/25779

De Jong-Gierveld, J., \& Kamphuls, F. (1985). The development of a Rasch-type loneliness scale. Applied psychological measurement, 9(3), 289-299.

De Jong-Gierveld, J., \& Tilburg, T. V. (2006). A 6-item scale for overall, emotional, and social loneliness: 


\section{Technology, loneliness, and social isolation}

Confirmatory tests on survey data. Research on aging, 28(5), 582-598.

Fakoya, O.A., McCorry, N.K., \& Donnelly, M. (2020). Loneliness and social isolation interventions for older adults: a systematic review of reviews. BMC Public Health, 20, 1-14. https://doi.org/10.1186/ s12889-020-251-6

Fields, J., Cemballi, A. G., Michalec, C., Uchida, D., Griffiths, K., Cardes, H., ... \& Lyles, C. R. (2020). Inhome technology training among socially isolated older adults: findings from the Tech Allies Program. Journal of Applied Gerontology, 1-11.

Fokkema, T., \& Knipscheer, K. (2007). Escape loneliness by going digital: A quantitative and qualitative evaluation of a Dutch experiment in using ECT to overcome loneliness among older adults. Aging \& Mental Health, 11(5), 496-504.

Gonzales, A. (2016) The contemporary US digital divide: from initial access to technology maintenance. Information, Communication \& Society, 19(2), 234-248. https://doi.org/10.1080/136911 8X.2015.1050438

Golden, J., Conroy, R. M., Bruce, I., Denihan, A., Greene, E., Kirby, M., \& Lawlor, B. A. (2009). Loneliness, social support networks, mood and wellbeing in community-dwelling elderly. International Journal of Geriatric Psychiatry: A journal of the psychiatry of late life and allied sciences, 24(7), 694-700.

Goumopoulos, C., Papa, I., \& Stavrianos, A. (2017). Development and evaluation of a mobile application suite for enhancing the social inclusion and wellbeing of seniors. Informatics, 4(3), 1-27.

Grenade, L. \& Boldy, D. (2008). Social isolation and loneliness among older people: Issues and future challenges in community and residential settings. Australian Health Review, 32(3), 468-478.

Herrera, M. S., Elgueta, R., Fernández, M. B., Giacoman, C., Leal, D., Marshall, P., Rubio, M., \& Bustamante, F. (2021). A longitudinal study monitoring the quality of life in a national cohort of older adults in Chile before and during the COVID-19 outbreak. BMC Geriatrics, 21(1), 143. https://doi. org/10.1186/s12877-021-02110-3

Holt-Lunstad, J. et al. (2015). Loneliness and social isolation as risk factors for mortality: A meta-analytic review. Perspectives on Psychological Science, 10(2), 227-237.

Infurna, F. (2021). Utilizing principles of life-span developmental psychology to study the complexities of resilience across the adult lifespan, The Gerontologist, 61(6): 807-818. https://doi.org/10.1093/geront/ gnab086

Joanna Briggs Institute (2011). Joanna Briggs Institute Reviewers' Manual: 2011 Edition. Adelaide, SA. Retrieved from : http://joannabriggs.org/assets/ docs/sumari/ReviewersManual-2011.pdf.

Kahlbaugh, P. E., Sperandio, A. J., Carlson, A. L., \& Hauselt, J. (2011). Effects of playing Wii on wellbeing in the elderly: Physical activity, loneliness, and mood. Activities, Adaptation \& Aging, 35(4), 331-344.

Kotwal, A. A., Holt-Lunstad, J., Newmark, R. L., Cenzer, I., Smith, A. K., Covinsky, K. E., Escueta, D. P., Lee, J. M., \& Perissinotto, C. M. (2021). Social isola- tion and loneliness among San Francisco Bay Area older adults during the COVID-19 Shelter-in-Place orders. Journal of the American Geriatrics Society, 69(1), 20-29. https://doi.org/10.1111/jgs.16865

Klasa, K., Galaitsi, S., Wister, A., \& Linkov, I. (2021). System models for resilience in gerontology: Application to the COVID-19 pandemic. BMC Geriatrics, 21(51), 1-12.

Knowles, L. M., Stelzer, E. M., Jovel, K. S., \& O'Connor, M. F. (2017). A pilot study of virtual support for grief: Feasibility, acceptability, and preliminary outcomes. Computers in Human Behavior, 73, 650-658.

Landerman, R., George, L.K., Campbell, R.T., Blazer, D.G. (1998). Alternative Models of the stress buffering hypothesis. American Journal of Community Psychology, 17, 625-642.

Larsson, E., Padyab, M., Larsson-Lund, M., \& Nilsson, I. (2016). Effects of a social internet-based intervention programme for older adults: An explorative randomised crossover study. British Journal of Occupational Therapy, 79(10), 629-636.

Leigh-Hunt, N., Bagguley, D., Bash, K., Turner, V., Turnbull, S., Valtorta, N., \& Caan, W. (2017). An overview of systematic reviews on the public health consequences of social isolation and loneliness. Public health, 152, 157-171.

Li, J., Xu, X., Pham, T. P., Theng, Y. L., Katajapuu, N., \& Luimula, M. (2017). Exergames designed for older adults: a pilot evaluation on psychosocial well-being. Games for health journal, 6(6), 371-378.

Luchetti, M., et al. (2020). The trajectory of loneliness in response to COVID-19. American Psychologist. Advance online publication http://dx.doi. org/10/1037/amp0000690

Morton, T. A., Wilson, N., Haslam, C., Birney, M., Kingston, R., \& McCloskey, L. G. (2018). Activating and guiding the engagement of seniors with online social networking: experimental findings from the AGES 2.0 project. Journal of aging and health, 30(1), 27-51.

Mullins, L. B., Skemp, L., Reed, D., \& Emerson, M. (2020). Internet programming to reduce loneliness and social isolation in aging. Research in gerontological nursing, 13(5), 233-242.

Munn, Z., Peters, M. D., Stern, C., Tufanaru, C., McArthur, A., \& Aromataris, E. (2018). Systematic review or scoping review? Guidance for authors when choosing between a systematic or scoping review approach. BMC medical research methodology, 18(1), 1-7.

National Seniors Council. (2014). Report on the Social Isolation of Seniors. Ottawa: Employment and Social Development Canada. Retrieved from: https:// www.canada.ca/en/national-seniors-council/programs/publications-reports/2014/social-isolationseniors.html

Neil-Sztramko, S. E., Coletta, G., Dobbins, M., \& Marr, S. (2020). Impact of the AGE-ON Tablet Training Program on Social Isolation, Loneliness, and Attitudes Toward Technology in Older Adults: SingleGroup Pre-Post Study. JMIR aging, 3(1), e18398.

Newall, N., McArthur, J., \& Menec, V. (2015). A longitudinal examination of social participation, loneliness, and use of physician and hospital services. Journal of Aging and Health, 27(3), 500-518. 


\section{Technology, loneliness, and social isolation}

Newall N., and Menec, V. (2019). Loneliness and social isolation of older adults: Why it is important to examine these social aspects together. Journal of Social and Personal Relationships, 36(3), 925-939. https://doi.org/10.1177/0265407517749045

Nicholson, N.E. (2009). Social isolation in older adults: An evolutionary concept analysis. Journal of Advanced Nursing, 65(6), 1342-1352.

Shahid, Z., Kalayanamitra, R., McClafferty, B., Kepko, D., Ramgobin, D., Patel, R., Aggarwal, C. S., Vunnam, R., Sahu, N., Bhatt, D., Jones, K., Golamari, R., \& Jain, R. (2020). COVID-19 and older adults: What we know. Journal of the American Geriatrics Society, 68(5), 926-929. https://doi.org/10.1111/jgs.16472

Pew Research Center. (2017). Tech Adoption Climbs Among Older Adults. Washington, DC: Pew Research Center Retrieved from: https://www.pewresearch.org/topic/internet-technology/user-demographics/age-generations-tech/older-adults-tech/

Riley, W. T., Pilkonis, P., \& Cella, D. (2011). Application of the National Institutes of Health patient-reported outcomes measurement information system (PROMIS®) to mental health research. The journal of mental health policy and economics, 14(4), 201.

Slegers, K., Van Boxtel, M. P., \& Jolles, J. (2008). Effects of computer training and Internet usage on the well-being and quality of life of older adults: A randomized, controlled study. The journals of gerontology series B: Psychological sciences and social sciences, 63(3), P176-P184.

Smith, M. L., Steinman, L. E., \& Casey, E. A. (2020). Combatting Social Isolation Among Older Adults in a Time of Physical Distancing: The COVID-19 Social Connectivity Paradox. Frontiers in Public Health, 8, 403. https://doi-org.proxy.lib.sfu. ca/10.3389/fpubh.2020.00403

Stolz, E., Mayerl, H., \& Freidl, W. (2021). The impact of COVID-19 restriction measures on loneliness among older adults in Austria. European journal of public health, 31(1), 44-49. https://doi.org/10.1093/ eurpub/ckaa238.

Strutt, P. A., Johnco, C. J., Chen, J., Muir, C., Maurice, O., Dawes, P., Siette, J., Botelho Dias, C., Hillebrandt, H., \& Wuthrich, V. M. (2021). Stress and Coping in Older Australians During COVID-19: Health, Service Utilization, Grandparenting, and Technology Use. Clinical Gerontologist, 1-13. https://doi.org/1 0.1080/07317115.2021.1884158

Tkatch, R., Wu, L., MacLeod, S., Ungar, R., Albright, L., Russell, D., ... \& Yeh, C. S. (2020). Reducing loneliness and improving well-being among older adults with animatronic pets. Aging \& mental health, 1-7.

Tomasino, K. N., Lattie, E. G., Ho, J., Palac, H. L., Kaiser, S. M., \& Mohr, D. C. (2017). Harnessing peer support in an online intervention for older adults with depression. The American Journal of Geriatric Psychiatry, 25(10), 1109-1119.

Toepoel, V. (2013). Ageing, leisure, and social connectedness: How could leisure help reduce social isolation of older people? Social Indicators Research, 113(1), 355-372.

Valtorta, N., Kanaan, M., Gilbody S., \& Hanratty, B. (2016). Loneliness, social isolation and social relationships: what are we measuring? A novel framework for classifying and comparing tools. BMJOpen , 6:e010799. https://doi.org/10.1136/bmjopen-2015-010799

van der Heide, L. A., Willems, C. G., Spreeuwenberg, M. D., Rietman, J., \& de Witte, L. P. (2012). Implementation of CareTV in care for the elderly: the effects on feelings of loneliness and safety and future challenges. Technology and Disability, 24(4), 283-291.

Victor, C.R., Scambler, S.J., Bowling, A.N.N., et al. (2005). The prevalence of, and risk factors for, loneliness in later life: a survey of older people in Great Britain. Ageing \& Society, 25(5), 357-375.

Weldrick, R., and Grenier, A. (2018). Social isolation in later life: Extending the conversation. Canadian Journal on Aging, 37(1): 76-83.

Windsor, R. (2015). Evaluation of Health Promotion and Disease Prevention Programs. NY: Oxford University Press.

Wister, A., Levasseur, M., Griffiths, L., \& Fyffe, I. (2015). Estimating multiple morbidity disease burden among older persons: A convergent construct validity study to discriminate between six chronic illness measures, CCHS 2008/09. Biomedical Central Geriatrics, 15(12), 1-12. https://doi.org/10.1186/ s12877-015-0001-8

Wister, A., Coatta, K., Schuurman, N., Lear, S., Rosin, M. \& MacKey, D. (2016). A Lifecourse model of multimorbidity resilience: Theoretical and research developments. International Journal of Aging \& Human Development, 82(24), 290-313. https://doi.org /10.1177/0 091415016641686

Wister, A., Menec, V., \& Mugford, G. (2018). Loneliness, social isolation, and social engagement. In P. Raina, C. Wolfson, S. Kirkland, \& L. Griffith (Eds.), The Canadian Longitudinal Study on Aging (CLSA) Report on Health and Aging in Canada (pp. 56-73). Canadian Longitudinal Study on Aging: McMaster University. www.clsa-elcv.ca/CLSAReport.

Wister, A., Lear, S., Schrumann, N., MacKey, D., Mitchell, B., Cosco, T. \& Fyffe, I. (2018). Development and validation of a multi-domain multimorbidity resilience index for an older population: Results from the baseline Canadian Longitudinal Study on Aging. Biomedical Central (BMC) Geriatrics, 18(170), 1-1 https: //doi.org /10.1186/s12877- 018 - 0851-y

Wister, A., Cosco, T., Mitchell, B. Menec, V. \& Fyffe, I. (2019). Development and concurrent validity of a composite social isolation index for older adults using the Canadian Longitudinal Study on Aging. Canadian Journal on Aging, 38(2), 180-192. https:// doi.org/10.1017/S0714980818000612

Wister, A.V. \& Cosco, T. (Eds). (2020). Resilience and Aging: Emerging Science and Future Possibilities. New York, NY.: Springer.

Wister, A. \& Speechley, M. (2020). COVID-19: Pandemic risk, resilience and possibilities for aging research. Canadian Journal on Aging, 39(3), 344-347.

Wister, A., Fyffe, I., \& O'Dea, E. (2021). Technological interventions for loneliness and social isolation among older adults: A scoping review protocol. Systematic Reviews, 10(217), 1-7.

Wister, A.V. \& Kadowaki, L. for the Federal/Provincial/ Territorial (FPT) Forum of Ministers Responsible for Seniors. (2021). Social Isolation Among Older 
Adults During the Pandemic. Employment and Social Development Canada. [forthcoming]

Woodward, A. T., Freddolino, P. P., Blaschke-Thompson, C. M., Wishart, D. J., Bakk, L., Kobayashi, R., \& Tupper, C. (2011). Technology and aging project: training outcomes and efficacy from a randomized field trial. Ageing International, 36(1), 46-65.

Woodward, A. T., Freddolino, P. P., Wishart, D. J., Bakk, L., Kobayashi, R. I. E., Tupper, C., ... \& BlaschkeThompson, C. M. (2013). Outcomes from a peer tutor model for teaching technology to older adults. Ageing \& Society, 33(8), 1315-1338.

Yu, R., Ellison, N., McCammon, R., \& Langa, K. (2016)
Mapping the two levels of digital divide: Internet access and social network site adoption among older adults in the USA. Information, Communication \& Society, 19(10), 1445-1464, https://doi.org/1 0.1080/1369118X.2015.1109695

Xie, B., Charness, N., Fingerman, K., Kaye, J., Kim, M. T., \& Khurshid, A. (2020). When going digital becomes a necessity: ensuring older adults' needs for information, services, and social inclusion during COVID-19. Journal of Aging \& Social Policy, 32(45), 460-470. https://doi-org.proxy.lib.sfu.ca/10.108 o/08959420.2020.1771237

\section{ApPendix I: SeARCh Strategy PyschINFO (EBSCO)}

("Aging" OR "older adults" OR elderly ) AND ( DE technology OR technology OR DE computers OR computers OR DE "information communication technology" OR DE internet OR internet OR robot ) AND ( DE loneliness OR loneliness OR DE "social isolation" OR "social isolation" ) AND ( intervention OR program OR evaluation OR trial ); Anytime-September 2020; English $=139$ results

\section{Ageline (EBSCO)}

( DE technology OR technology OR DE computers OR computers OR DE internet OR internet OR "information and communication technology" OR robot ) AND ( DE loneliness OR loneliness OR DE "social isolation" OR "social isolation" ) AND ( intervention OR program OR evaluation OR trial ); Anytime- September 2020, English $=66$ results

\section{Academic Search Premier (EBSCO)}

(DE "older people" OR "older adults" OR "elderly") AND ( DE technology OR technology OR DE computers OR computers OR DE "information communication technology" OR DE internet OR internet OR robot) AND (DE loneliness OR loneliness OR DE "social isolation" OR "social isolation" ) AND ( intervention OR program OR evaluation OR trial )

Anytime-September 2020, English

$=177$ results

\section{Medline (EBSCO)}

Search: ( MM Aged [MeSH term] OR MM "Aged, 80 and over"[MeSH term] OR TX "older adults" OR TX elderly ) AND ( MM technology [MeSH term] or technology [All Fields] OR MM "UserComputer Interface" OR TX Computers OR MM internet [MeSH term] OR internet [All Fields] OR MM "computers, handheld" [MeSH term] OR ICT [All Fields] OR Robot [All Fields] ) AND ( $\mathrm{MM}$ loneliness [MeSH term] OR loneliness [All
Fields] OR MM "social isolation" [MeSH term] OR social isolation [All Fields] ) AND ( intervention [All Fields] OR program [All Fields] OR evaluation [All Fields] OR trial [All Fields])

Anytime-September 2020, English

$=253$ results

\section{Global Health (EBSCO)}

(DE elderly OR" older adults") AND (DE technology OR technology OR DE computers OR computers OR DE internet OR internet OR "information and communication technology" OR robot ) AND ( loneliness OR "social isolation" ) AND ( intervention OR program OR evaluation OR trial ); Anytime-September 2020, English $=11$ Results

\section{Web of Science}

((older adults OR elderly) AND (technology OR computers OR internet OR "information and communication technology" OR robot) AND (loneliness or "social isolation") AND (intervention OR program OR evaluation OR trial)); Anytime-September 2020; English

$=323$ results

\section{Clinical Trials.gov}

Keywords: condition or disease: loneliness

Other terms: technology, older adults

Study type: All studies

Study results: Studies with results

Age: Adult (18-64) and older adults (65+)

Results first posted: Anytime- September2020

$=9$ results

Keywords: social isolation

Other terms: technology, older adults: 20 results

Study type: All studies

Study results: Studies with results

Age: Adult (18-64) and older adults (65+)

Results first posted: Anytime- September 2020

$=14$

$=23$ results total 